\title{
THE RANGE OF INVARIANT MEANS ON LOCALLY COMPACT GROUPS AND SEMIGROUPS
}

\author{
ROY C. SNELL ${ }^{1}$
}

\begin{abstract}
This paper extends the results of Granirer and Chou concerning the range of a left invariant mean on a discrete semigroup to the case when $S$ is any Borel subsemigroup of a locally compact group.
\end{abstract}

0. Introduction. Granirer has shown in [2] that for an infinite, right cancellation, left amenable discrete semigroup $S$, other than what he calls an " $A B$ group", there exists a nested family of left almost convergent subsets of $S$ on which any left invariant mean (LIM) attains all values of the closed interval $[0,1]$. That is, there exists a family $\{A(t) \mid t \in[0,1]\}$ of subsets of $S$ for which

(i) $s<t$ implies $A(s) \subseteq A(t)$, and

(ii) $\varphi\left(\chi_{A(t)}\right)=t$ for any $\operatorname{LIM} \varphi$ on $m(S)$.

Chou, in [1], partially extended this result to the case when $S$ is a group and obtained the following theorem thereby proving a conjecture made in [2].

THEOREM (CHOU). If $S$ is an infinite right cancellation left amenable semigroup then the range of each LIM on $m(S)$ is the whole $[0,1]$ interval.

In this paper we extend these results to locally compact topological groups and obtain the following main theorems:

THEOREM A. Let $S$ be an infinite Borel subsemigroup of positive Haar measure in a locally compact group. If $\varphi$ is a LIM on $L^{\infty}(S)$ then there exists a nested family $\{A(t) \mid t \in[0,1]\}$ of Borel subsets of $S$ such that $\varphi(A(t))=t$ for all $t \in[0,1]$.

THEOREM B. Let $S$ be an infinite Borel subsemigroup in a locally compact group and let $A$ denote the algebra of bounded Borel measurable

Received by the editors March 7, 1972.

AMS (MOS) subject classifications (1969). Primary 2875, 2220; Secondary 4250, 4655 .

${ }^{1}$ The results in this paper constitute a portion of the author's Ph.D. thesis written under the direction of Dr. E. A. Granirer at the University of British Columbia. The author wishes to thank Professor Granirer for his aid and advice and as well the Canada Council for its financial support.

(c) American Mathematical Society 1973 
functions on $S$. If $\varphi$ is a LIM on $A$ then there exists a nested family $\{A(t) \mid t \in[0,1]\}$ of Borel subsets of $S$ such that $\varphi(A(t))=t$ for all $t \in[0,1]$.

These theorems improve Chou's results even for the case that $G$ is discrete, by providing a nested family of subsets on which a given LIM attains all values in the $[0,1]$ interval. They also supply answers concerning the range of a LIM on such frequently encountered spaces as $L^{\infty}(R)$ ( $R$ the additive reals with the usual topology) to which Granirer's and Chou's results do not apply.

However, the nested sets obtained in these theorems depend heavily on the mean $\varphi$ being considered and hence are not left almost convergent as are those obtained by Granirer for discrete semigroups.

We have been able to show that if $G$ is abelian and compactly generated, there exists a nested family of left almost convergent Borel sets with the desired property (manuscript in preparation) but we do not know how to obtain such a family for arbitrary locally compact groups.

The results in the body of this paper are somewhat more general than the ones quoted in this introduction.

1. Preliminaries. By a function algebra on a nonvoid set $S$ we mean a sup norm closed, point separating algebra of bounded real-valued functions on $S$ containing the constants. If $(X, \mathscr{S}, \mu)$ is a measure space we denote by $L^{\infty}(X)$ the algebra of bounded, real-valued, $\mathscr{S}$-measurable functions on $X$ with the essential sup norm (so $L^{\infty}(X)$ is not strictly speaking a function algebra).

If $S$ is a semigroup we say that a function algebra $A$ on $S$ is left invariant if $L_{t} f \in A$ for all $t \in S, f \in A$ where $L_{t} f(s)=f(t s)$ for all $s \in S$. An element $\varphi$ in $A^{*}$ is called a mean if $\|\varphi\|=1$ and $\varphi(f) \geqq 0$ whenever $f \geqq 0$ in $A$. $\varphi$ is said to be a left invariant mean (LIM) on $A$ if $\varphi\left(L_{t} f\right)=\varphi(f)$ for all $t \in S$ and $f \in A$. If $B \subseteq S$ is such that $\chi_{B} \in A$ (where $\chi_{B}$ denotes the characteristic function of the set $B$ ) then, for convenience of notation, we will often refer to $\varphi(B)$ rather than $\varphi\left(\chi_{B}\right)$ if no confusion will result. A function $f \in A$ is called left almost convergent if $\varphi(f)$ is the same value for any LIM $\varphi$ on $A$.

If $A$ is a function algebra (or $L^{\infty}(X)$ for a measure space $(X, \mathscr{S}, \mu)$ ) then the structure space $\Delta(A)$ of $A$ is the set of all multiplicative means on $A$ (i.e. those means $\varphi$ for which $\varphi(f \cdot g)=\varphi(f) \cdot \varphi(g)$ for all $f, g \in A$ ) and is equipped with the $w^{*}$ topology as a subset of $A^{*}$. Under this topology $\Delta(A)$ is a compact Hausdorff space and $A$ is homeomorphic to the continuous functions on $\Delta(A)$ under the mapping

$$
f \rightarrow \hat{f} \text { where } \hat{f}(\lambda)=\lambda(f) \text { for all } \lambda \in \Delta(A)
$$

(see [5, p. 479] for details). 
If $S$ is a topological space we will denote by $B(S)$ the Borel subsets of $S$ (i.e. the smallest $\sigma$-field of subsets containing the open sets). A subsemigroup $T$ of $S$ will be called a Borel subsemigroup if $T \in B(S)$.

If $\mathscr{B}$ is a $\sigma$-field of subsets of a semigroup $S$ then $\mathscr{B}$ is called left invariant if $t^{-1} B \in \mathscr{B}$ for all $t \in S$ and $B \in \mathscr{B}$ (where $t^{-1} B=\{s \in S \mid t s \in B\}$ ). Note that if. $\mathscr{B}$ is a left invariant $\sigma$-field and $A$ is the algebra of bounded $\mathscr{B}$ measurable functions then $A$ is a left invariant function algebra.

If $S$ is a topological semigroup, the algebra of left uniformly continuous, bounded functions on $S$, denoted by $\operatorname{LUC}(S)$ consists of all bounded continuous functions $f$ on $S$ with the property that

$$
\lim \left\|L_{s_{\alpha}} f-L_{s_{0}} f\right\|=0 \quad \text { whenever } s_{\alpha} \rightarrow s_{0} \text { in } S .
$$

Let $\mathscr{B}$ be a $\sigma$-field of subsets of a nonvoid set $S$. If $A$ is the algebra of bounded $\mathscr{B}$-measurable functions on $S$ then the topology on $\Delta(A)$ can be characterized as follows:

For $B \in \mathscr{B}$ let $U_{B 3}=\{\psi \in \Delta(A) \mid \psi(B)=1\}$. Then $U_{B}$ is open-closed in $\Delta(A)$ and $\left\{U_{B} \mid B \in \mathscr{B}\right\}$ is a base for the topology on $\Delta(A)$. (This can be easily shown using the facts that the simple functions on $\mathscr{B}$ are dense in $A$ and that each $\psi \in \Delta(A)$ is multiplicative which implies that $\psi(B)=0$ or 1 for all $B \in \mathscr{B}$.) In a similar fashion it can be shown that, for a measure space $(X, \mathscr{S}, \mu),\left\{U_{B} \mid B \in \mathscr{S}\right\}$ is a base of open-closed sets for the topology on $\Delta\left(L^{\infty}(X)\right)$.

2. Probability measures on $\Delta(A)$. If $\varphi$ is a mean on a function algebra $A$ then, since $A$ is isometrically isomorphic to the continuous functions on the compact, Hausdorff space $\Delta(A)$, there exists a regular probability measure $\mu_{\varphi}$ on $\Delta(A)$ such that

$$
\int_{\Delta(A)} \hat{f} d \mu_{\varphi}=\varphi(f) \text { for all } f \in A .
$$

Note that when $A$ is the algebra of bounded functions measurable with respect to a $\sigma$-field $\mathscr{B}$ then we have $\mu_{\varphi}\left(U_{B}\right)=\varphi(B)$ for all $B \in \mathscr{B}$.

Let $X$ be locally compact and Hausdorff and $\mu$ a regular probability measure (as in [5]) on $B(X)$. If $\mu$ is continuous, i.e. $\mu(\{x\})=0$ for all $x \in X$, then by [5, p. 132], for all $B \in B(X)$ we have $\left\{\mu\left(B^{\prime}\right) \mid B^{\prime} \in B(X), B^{\prime} \subset B\right\}=$ $[0, \mu(B)]$. Combining an idea of Chou in [1] and an idea of Granirer in [2] we now prove the following lemma which will be the key tool used in obtaining the main results of this paper.

LEMMA 2.1A. Let $S$ be a nonempty set. $\mathscr{B}$ a $\sigma$-field of subsets of $S$ and $A$ the algebra of bounded, $\mathscr{B}$-measurable functions on $S$. Let $\varphi$ be a mean on $A$ for which the corresponding probability measure $\mu_{\varphi}$ is continuous. 
Then there exists a family $\{A(t) \mid t \in[0,1]\}$ in $\mathscr{B}$ such that

(i) $s<t$ implies $A(s) \subset A(t)$, and

(ii) $\varphi(A(t))=t$ for all $t \in[0,1]$.

REMARKS. In particular for the range of $\varphi$ we have $\{\varphi(B) \mid B \in \mathscr{B}\}$ is all of the interval $[0,1]$. It should also be noted that the sets $A(t)$ obtained depend heavily on the mean $\varphi$ being considered.

Proof. Let $A_{0}$ and $A_{1} \in \mathscr{B}$ with $A_{0} \subset A_{1}$ and let $\lambda \in\left[\varphi\left(A_{0}\right), \varphi\left(A_{1}\right)\right]$. Since $\left(\lambda-\varphi\left(A_{0}\right)\right) \in\left[0, \mu_{\varphi}\left(U_{A_{1}} \sim U_{A_{0}}\right)\right]=\left[0, \varphi\left(A_{1}\right)-\varphi\left(A_{0}\right)\right]$ there exists a Borel set $B$ in $\Delta(A)$ with $B \subset U_{A_{1}} \sim U_{A_{0}}$ and $\mu_{\varphi}(B)=\lambda-\varphi\left(A_{0}\right)$. Letting $E=U_{A_{0}} \cup B$ we have $U_{A_{0}} \subset E \subset U_{A_{1}}$ and $\mu_{\varphi}(E)=\lambda$.

Given $\varepsilon>0$, by the regularity of $\mu_{\varphi}$ and using the facts that $U_{A_{0}}$ is compact and $U_{A_{1}}$ is open, we can find a compact $E_{1}$ and an open $E_{2}$ with $U_{A_{0}} \subset E_{1} \subset E \subset E_{2} \subset U_{A_{1}}$ and $\mu_{\varphi}\left(E_{2} \sim E_{1}\right)<\varepsilon$. Since $E_{1}$ is compact and $E_{2}$ open, using the fact that $\left\{U_{B} \mid B \in \mathscr{B}\right\}$ is a base for the topology, we can find $B \in \mathscr{B}$ with $E_{1} \subset U_{B} \subset E_{2}$ so $|\varphi(B)-\lambda|<\varepsilon$. Since $A_{0} \subset B \subset A_{1}$ this implies $\left\{\varphi(B) \mid B \in \mathscr{B}, A_{0} \subset B \subset A_{1}\right\}$ is dense in [ $\left.\varphi\left(A_{0}\right), \varphi\left(A_{1}\right)\right]$.

Let $Q_{n}=\left\{k / 2^{n} \mid k=0,1, \cdots, 2^{n}\right\}$ and $Q=\bigcup_{n=1}^{\infty} Q_{n}$. For $t \in Q$ we want to define $A(t) \in \mathscr{B}$ such that $\{A(t) \mid t \in Q\}$ satisfies (i) and (ii). We proceed inductively by defining $A(0)=\varnothing, A(1)=S$ and assuming that we have $\left\{A(t) \mid t \in Q_{n}\right\}$ satisfying the required conditions. For $t \in Q_{n+1} \cap Q_{n}$ we let $A^{\prime}(t)=A(t)$. If $t \in Q_{n+1} \sim Q_{n}$ let $t_{1}, t_{2} \in Q_{n}$ be the maximum and minimum elements of $Q_{n}$ for which $t_{1}<t<t_{2}$. Choose sequences $\alpha_{n} \uparrow t$ and $\beta_{n} \downarrow t$ in $\left(t_{1}, t_{2}\right)$. Since $\left\{\varphi(B) \mid B \in \mathscr{B}, A\left(t_{1}\right) \subset B \subset A\left(t_{2}\right)\right\}$ is dense in $\left[t_{1}, t_{2}\right]$ we can find $C_{1} \in \mathscr{B}$ with $A\left(t_{1}\right) \subset C_{1} \subset A\left(t_{2}\right)$ and $\beta_{2}<\varphi\left(C_{1}\right)<\beta_{1}$. Since $\left(\alpha_{1}, \alpha_{2}\right) \subset$ $\left[t_{1}, \varphi\left(C_{1}\right)\right]$ we can find $D_{1} \in \mathscr{B}$ with $A\left(t_{1}\right) \subset D_{1} \subset C_{1}$ and $\alpha_{1}<\varphi\left(D_{1}\right)<\alpha_{2}$. Since $\left(\beta_{3}, \beta_{2}\right) \subset\left[\varphi\left(D_{1}\right), \varphi\left(C_{1}\right)\right]$ there exists $C_{2} \in \mathscr{B}$ with $D_{1} \subset C_{2} \subset C_{1}$ and $\beta_{3}<\varphi\left(C_{2}\right)<\beta_{2}$. Continuing in this manner we obtain sequences $C_{i} \downarrow$ and $D_{i} \uparrow$ in $\mathscr{B}$ with $A\left(t_{1}\right) \subset D_{i} \subset C_{j} \subset A\left(t_{2}\right)$ for all $i, j$ and such that $\alpha_{i}<\varphi\left(D_{i}\right)<$ $\alpha_{i+1}, \beta_{i+1}<\varphi\left(C_{i}\right)<\beta_{i}$ for all $i$. If we let $A^{\prime}(t)=\bigcup_{i=1}^{\infty} D_{i} \in \mathscr{B}$ we have $D_{i} \subset A^{\prime}(t) \subset C_{j}$ for all $i, j$ which implies $\varphi\left(A^{\prime}(t)\right)=t$ and also $A^{\prime}\left(t_{1}\right) \subset$ $A^{\prime}(t) \subset A^{\prime}\left(t_{2}\right)$. In this manner we obtain a new collection $\left\{A^{\prime}(t) \mid t \in Q_{n+1}\right\}$ in $\mathscr{B}$ which satisfies (i) and (ii) and extends the collection $\left\{A(t) \mid t \in Q_{n}\right\}$. By induction we have a collection $\{A(t) \mid t \in Q\}$ with $\varphi(A(t))=t$ for all $t \in Q$ and if $t_{1}, t_{2} \in Q$ with $t_{1}<t_{2}$ then $t_{1}, t_{2} \in Q_{n}$ for some $n$ so $A\left(t_{1}\right) \subset A\left(t_{2}\right)$.

For $t \in[0,1]$ let $A(t)=\bigcap\{A(s) \mid s \in Q, t \leqq s\}$. Since $Q$ is countable, $A(t) \in \mathscr{B}$ and if $t_{0}<t_{1}$ we have $A\left(t_{0}\right) \subset A\left(t_{1}\right)$. Also since $s_{1}=\varphi\left(A\left(s_{1}\right)\right) \leqq$ $\varphi(A(t)) \leqq \varphi\left(A\left(s_{2}\right)\right)=s_{2}$ for all $s_{1}, s_{2} \in Q$ with $s_{1} \leqq t \leqq s_{2}$, the density of $Q$ in $[0,1]$ implies $\varphi(A(t))=t$ so the collection satisfies the required conditions.

The same argument gives us, as well, the following

LEMMA 2.1B. Let $(X, \mathscr{S}, \mu)$ be a measure space. Let $\varphi$ be a mean on 
$L^{\infty}(X)$ for which the corresponding probability measure $\mu_{\varphi}$ on $\Delta\left(L^{\infty}(X)\right)$ is continuous. Then there exists a family $\{A(t) \mid t \in[0,1]\}$ in $\mathscr{S}$ such that

(i) $s<t$ implies $A(s) \subset A(t)$, and

(ii) $\varphi(A(t))=t$ for all $t \in[0,1]$.

3. Orbits in $\Delta(A)$. Let $A$ be a left invariant function algebra on a semigroup $S$. If $\psi \in \Delta(A)$ then the left orbit of $\psi$

$$
O(\psi)=\left\{L_{t}^{*} \psi \mid t \in S\right\}
$$

is a subset of $\Delta(A)$ where $L_{t}^{*}$ denotes the adjoint of the left translation operator $L_{t}$. In order to apply Lemma $2.1 \mathrm{~A}$ we will be interested in the case where $O(\psi)$ is infinite for all $\psi \in \Delta(A)$. In this case any LIM on $A$ will have the property that $\mu_{\varphi}$ is a continuous measure on $\Delta(A)$.

LeMma 3.1A. Let $S$ be an infinite Borel subsemigroup of a locally compact group $G$ and $A$ the algebra of bounded Borel measurable functions on $S$. Then $O(\psi)$ is infinite for all $\psi \in \Delta(A)$.

Proof. First note that for $\psi_{1} \neq \psi_{2}$ in $\Delta(A)$ we can find $B \in B(S)$ with $\psi_{1}(B) \neq \psi_{2}(B)$. Let $C=t B$ where $t \in S$. Since $S$ is a Borel set in $G$ we have $B(S)=\{B \in B(G) \mid B \subset S\}$ and since $t \cdot B(G)$ is contained in $B(G)$ for all $t \in G$, this implies that $C \in B(S)$. Thus $\chi_{C} \in A$ with $L_{t} \chi_{C}=\chi_{B}$ so

$$
L_{t}^{*} \psi_{1}(C)=\psi_{1}\left(L_{t} \chi_{C}\right)=\psi_{1}(B) \neq \psi_{2}(B)=\psi_{2}\left(L_{t} \chi_{C^{\prime}}\right)=L_{t}^{*} \psi_{2}(C)
$$

and the mapping $L_{t}^{*}: \Delta(A) \rightarrow \Delta(A)$ is one-to-one.

If $O(\psi)$ is finite for some $\psi \in \Delta(A)$ then we can find $t_{1}, t_{2}, \cdots, t_{n} \in S$ such that

(i) $L_{t_{i}}^{*} \psi \neq L_{t_{j}}^{*} \psi$ for $i \neq j$, and

(ii) for any $t \in S$ we have $L_{t}^{*} \psi=L_{t_{i}}^{*} \psi$ for some $i$.

If we set $\varphi=\left(\sum_{i=1}^{n} L_{t_{i}}^{*} \psi\right) / n$ it is easily checked that $\varphi$ is a LIM on $A$ and so, by restriction, also a $\operatorname{LIM}$ on $\operatorname{LUC}(S)$ which is a closed subspace of $A$. However Theorem 3 of [4] shows that for infinite $S, \operatorname{LUC}(S)$ has no LIM in the convex hull of the multiplicative means. This contradiction shows that $O(\psi)$ must be infinite for all $\psi \in \Delta(A)$.

The same argument can be used to prove as well

Lemma 3.1B. Let $S$ be an infinite Borel subsemigroup of positive Haar measure in a locally compact group $G$. Then $O(\psi)$ is infinite for all $\psi \in \Delta\left(L^{\infty}(S)\right)$

\section{Main results.}

THEOREM 4.1. Let $S$ be a semigroup, $\mathscr{B}$ a left invariant $\sigma$-field of subsets of $S$ and $A$ the algebra of bounded $\mathscr{B}$-measurable functions on $S$. 
If $O(\psi)$ is infinite for all $\psi \in \Delta(A)$ then for any LIM $\varphi$ on $A$ there exists a family $\{A(t) \mid t \in[0,1]\}$ in $\mathscr{B}$ such that

(i) $s<t$ implies $A(s) \subset A(t)$, and

(ii) $\varphi(A(t))=t$ for all $t \in[0,1]$.

Proof. This result follows from Lemma 2.1A if we can show that $\mu_{\varphi}$ is continuous. Choose $\psi \in \Delta(A)$ and $t \in S$. Note that if $L_{t}^{*} \psi \in U_{B B}=$ $\{\omega \in \Delta(A) \mid \omega(B)=1\}$ where $B \in \mathscr{B}$ then $\psi \in U_{t^{-1} B}$ and since

$$
\mu_{\varphi}\left(U_{B}\right)=\varphi(B)=\varphi\left(L_{t} \chi_{B}\right)=\varphi\left(\chi_{t^{-1} B}\right)=\mu_{\varphi}\left(U_{t^{-3} B}\right)
$$

we have $\mu_{\varphi}(\{\psi\}) \leqq \mu_{\varphi}\left(U_{B}\right)$ whenever $L_{t}^{*} \psi \in U_{B}$. The regularity of $\mu_{\varphi}$ and the fact that $\left\{U_{B} \mid B \in \mathscr{B}\right\}$ is a base for the open sets in $\Delta(A)$ implies that $\mu_{\varphi}(\{\psi\}) \leqq \mu_{\varphi}\left(\left\{L_{t}^{*} \psi\right\}\right)$.

Since $O(\psi)$ is infinite, for any integer $n$ we can find $t_{1}, t_{2}, \cdots, t_{n}$ in $S$ with $L_{t_{i}}^{*} \psi \neq L_{t_{j}}^{*} \psi$ for $i \neq j$. Thus

$$
1=\mu_{\varphi}(\Delta(A)) \geqq \mu_{\varphi}\left(\bigcup_{i=1}^{n}\left\{L_{t_{i}}^{*} \psi\right\}\right)=\sum_{i=1}^{n} \mu_{\varphi}\left(\left\{L_{f_{i}}^{*} \psi\right\}\right) \geqq n \cdot \mu_{\varphi}(\{\psi\})
$$

and since $n$ is arbitrary, this implies that $\mu_{\varphi}(\{\psi\})=0$ so $\mu_{\varphi}$ is continuous.

Combining this result with Lemma 3.1A we obtain

THEOREM 4.2A. Let $S$ be an infinite Borel subsemigroup of a locally compact group $G$. If $A$ is the algebra of bounded Borel measurable functions on $S$ and $\varphi$ is a LIM on $A$ then there exists a family $\{A(t) \mid t \in[0,1]\}$ of Borel subsets of $S$ such that

(i) $s<t$ implies $A(s) \subset A(t)$ and

(ii) $\varphi(A(t))=t$ for all $t \in[0,1]$.

As a corollary to Theorem $4.2 \mathrm{~A}$ we obtain a slightly stronger version of Chou's result in [1].

COROLlary 4.2.1. Let $G$ be an infinite discrete group and let $q$ be a $L I M$ on $m(G)$ (the bounded real valued functions on $G$ with the sup norm). Then there exists a family $\{A(t) \mid t \in[0,1]\}$ of subsets of $G$ such that

(i) $s<t$ implies $A(s) \subset A(t)$, and

(ii) $\varphi(A(t))=t$ for all $t \in[0,1]$.

The method of proof used in Theorem 4.1 will also work in the case where $S$ is a Borel subsemigroup of positive Haar measure in a locally compact group and $A=L^{\infty}(S)$ (strictly speaking not a function algebra). Combining this with Lemma 3.1B we obtain

THEOREM 4.2B. Let $S$ be an infinite Borel subsemigroup of positive Haar measure in a locally compact group $G$. If $\varphi$ is a LIM on $L^{\infty}(S)$ then there 
exists a family $\{A(t) \mid t \in[0,1]\}$ of Borel subsets of $S$ such that

(i) $s<t$ implies $A(s)=A(t)$, and

(ii) $\varphi(A(t))=t$ for all $t \in[0,1]$.

5. Comments. Let $S$ be an infinite, left amenable, right cancellation discrete semigroup and $A=m(S)$ (the bounded real valued functions on $S$ ). If $S$ contains no element of infinite order then Lemma 4 of [2] shows that $S$ is a group so, given any LIM $\varphi$ on $A$, Theorem $4.2 \mathrm{~A}$ gives us a nested family of subsets of $S$ on which $\varphi$ attains all values in the interval $[0,1]$. On the other hand if $t \in S$ has infinite order, then for any integer $n$, Lemma 1 of [3] gives us a partition of $S$ into disjoint subsets $A_{1}, A_{2}, \cdots$, $A_{n}$ which are such that $t A_{i} \subset A_{i+1}$ for $1 \leqq i \leqq n-1$ and $t A_{n} \subset A_{1}$. If $\psi \in \Delta(A)$ we can assume that $\psi \in U_{A_{1}}$ and if $0 \leqq k \leqq n-1$ we have $t^{k} A_{1} \subset A_{k+1}$ so $L_{t^{k}} \chi_{A_{k+1}} \geqq L_{t^{k}} \chi_{t^{k} A_{1}} \geqq \chi_{A_{1}}$ implies $1 \geqq \psi\left(L_{t^{k}} \dot{\chi}_{A_{k+1}}\right) \geqq \psi\left(A_{1}\right)=1$.

Thus $L_{t^{k}}^{*} \psi \in U_{A_{k+1}}$ for $k=0,1, \cdots, n-1$. But $U_{A_{i}} \cap U_{A_{j}}=\varnothing$ for $i \neq j$ so $\psi, L_{t}^{*} \psi, \cdots, L_{t^{(n-1)}}^{*} \psi$ is a set of $n$ distinct elements in $O(\psi)$. Since $n$ was arbitrary this implies that $O(\psi)$ is infinite for all $\psi \in \Delta(A)$ and Theorem 4.1 gives us, for any LIM $\varphi$ on $m(S)$, a nested family of subsets of $S$ on which $\varphi$ attains all values in $[0,1]$. This result is weaker than Theorem 3 in [2]. There Granirer is able to find such a nested family of left almost convergent sets (i.e. $\varphi(A(t))=t$ for any $\operatorname{LIM} \varphi$ on $m(S)$ ).

It would be interesting to determine whether the range of a LIM on $L^{\infty}(S)$ is attained on the left almost convergent Borel subsets of $S$, for any semigroup $S$.

\section{REFERENCES}

1. C. Chou, On a conjecture of E. Granirer concerning the range of an invariant mean, Proc. Amer. Math. Soc. 26 (1970), 105-107. MR 41 \#5519.

2. E. Granirer, On the range of an invariant mean, Trans. Amer. Math. Soc. 125 (1966), 384-394. MR 34 \#4390.

3. - Extremely amenable semigroups, Math. Scand. 17 (1965), 177-197. MR 33 \#5760.

4. E. Granirer and A. Lau, Invariant means on locally compact groups, Illinois J. Math. 15 (1971), 249-257.

5. E. Hewitt and K. A. Ross, Abstract harmonic analysis. Vol. 1 : Structure of topological groups. Integration theory, group representations, Die Grundlehren der math. Wissenschaften, Band 115, Academic Press, New York; Springer-Verlag, Berlin, 1963. MR 28 \#158.

Department of Mathematics, University of British Columbia, Vancouver, British Columbia, Canada

Current address: Department of Mathematics, Royal Roads Military College, Victoria, British Columbia, Canada 